\title{
Purple Urine Bags Syndrome masquerading as a bowel obstruction
}

\author{
Declan McDonnel1* and Andrew Aldridge \\ Conquest Hospital, St Leonards-on-Sea, East Sussex, UK
}

\section{Introduction}

A 79-year-old patient who had undergone radical cystectomy with ileal conduit formation in 2015 presented to the surgical assessment unit with a week-long history of abdominal distention and constipation and raised inflammatory markers. A CT was performed which attributed the cause of the apparent obstruction to a right inguinal hernia containing several bowel loops. However, this hernia was easily reducible and an alternative explanation for the presentation was sought. On closer inspection of the patient's abdomen, his urostomy bag was noticed to be purple in colour (Figure 1). A urine dip tested positive for leucocytes and nitrites, so the patient was treated with intravenous co-amoxiclav and fluid therapy. The patient's bowels started to work once his clinical condition improved. Further urine analysis revealed the $\mathrm{pH}$ to be acidic, an unusual finding in purple urine bag syndrome, and a culture grew Staphylococcus aureus.

\section{Background}

Dietary tryptophan is metabolised by gut flora to indole, which in turn is conjugated with a sulphate group by the liver. Bacteria producing indoxyl suphatase can proliferate in such environments and convert indoxyl sulphate to indigo and indirubicin, which are blue and red respectively, to give a purple appearance of the urine [1]. It is important to note that it is the reaction of the compounds with the plastic of the bag and catheter which give the characteristic appearance [2].

This case is important because Purple Urine Bag Syndrome (PUBS) is an unusual problem most commonly found in patients with long-

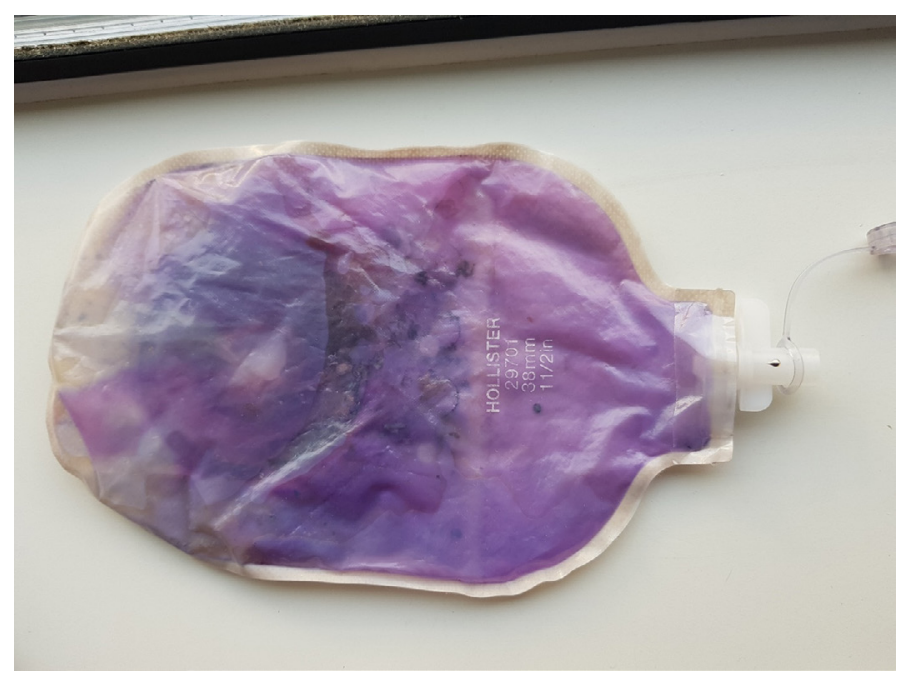

Figure 1. A purple urostomy bag term catheters in the presence of alkaline urine. This patient had an ileal conduit and acidic urine, which enabled Staphylococcus aureus to colonise the neobladder. Although unusual, Staph. species have been found in other cases of PUBS [3].

Another important feature of this case was the apparent obstruction due to the presence of a hernia containing bowel loops on the CT. It is worth highlighting that PUBS often occurs in conjunction with constipation. Although constipation will promote conversion of tryptophan to indole by gut flora, it is likely the paralytic ileus was precipitated by urosepsis given how quickly the problem resolved on appropriate antibiotic therapy. It is important that surgeons recognise that non-obstructed hernias and paralytic ileus due to infection can exist concomitantly and therefore, avoid unnecessary operations.

\section{Case presentation}

A 79-year-old patient presented with a seven-day history of increasing abdominal distention and constipation. A right inguinal hernia was noticed but this was easily reducible, and given the patient's surgical history, having undergone radical cystectomy with ileal conduit formation two years previously, an adhesional bowel obstruction was assumed. Due to the raised inflammatory markers (white cell count $27 \times 109 / \mathrm{L}, \mathrm{CRP} 272 \mathrm{mg} / \mathrm{L}$ ), concerns about a possible or impending perforation was made so an out of hours CT scan was requested. He also had a significant acute kidney injury (creatinine 248 from a baseline of $120 \mu \mathrm{mol} / \mathrm{L}$ ) which necessitated a non-contrast scan. This demonstrated dilated small bowel loops, particularly within the inguinal hernia, but a full assessment as limited due to the avoidance of contrast.

The patient was reviewed on the consultant's post-take ward round, and it was felt that the hernia was not causing a bowel obstruction. It was at this time the purple discolouration of the urostomy bag was noted and the possibility of Purple Urine Bag Syndrome (PUBS) was raised.

\section{Investigations}

Urinalysis was performed which showed evidence of infection, although the $\mathrm{pH}$ was acidic, which is not in keeping with the typical presentation of this clinical entity. However, the urine culture grew Staphylococcus aureus, which itself is not a typical organism of this condition.

${ }^{\star}$ Correspondence to: Declan McDonnell, Conquest Hospital, St Leonards-onSea, East Sussex, UK, E-mail: declan@doctors.org.uk

Received: May 17, 2019; Accepted: May 21, 2019; Published: May 25, 2019 


\section{Treatment}

The patient was treated with a broad-spectrum intravenous antibiotic, namely co-amoxiclav, and fluids. He had a good response to this, and his ileus started to resolve within 48 hours of starting the treatment.

\section{Outcome and follow-up}

This gentleman had a good response to intravenous antibiotics and fluids, with his urine no longer having the characteristic appearance (Figure 2). After a four-day admission, with his ileus having resolved and inflammatory markers returning to normal, this patient was discharged home on oral antibiotics. He has been advised to look for symptoms suggestive of an ileus or PUBS in the future.

\section{Discussion}

PUBS is a recognised problem usually apparent in alkaline urine in the presence of bacteria such as Escherichia coli or Proteus species [3]. It could be a useful clinical sign to aid diagnosis in patients presenting with urosepsis or other manifestations secondary to urinary tract infection.

\section{Learning points}

- The presence of purple urine should highlight the presence of particular bacterial colonisation in urine which could contribute to the patient's clinical condition.

- An ileus in the presence of a hernia containing bowel loops can mimic an obstructed hernia which could have resulted in an unnecessary operation.

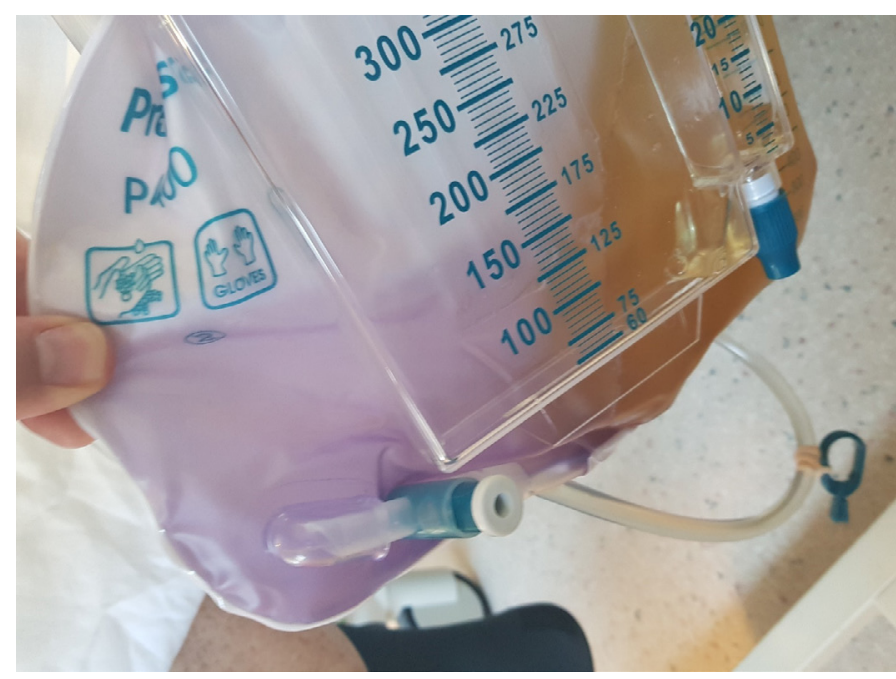

Figure 2. A catheter bag with purple precipitation and normal urine

- Patients with urostomies are likely to have neobladders colonised with organisms not usually associated with the urinary tract and should therefore have therapeutics adjusted accordingly.

\section{References}

1. Dealler SF, Hawkey PM, Millar MR (1988) Enzymatic degradation of urinary indoxyl sulfate by Providencia stuartii and Klebsiella pneumoniae causes the purple urine bag syndrome. J Clin Microbiol 26: 2152-2156. [Crossref]

2. Tan CK, Wu YP, Wu HY, Lai CC (2008) Purple urine bag syndrome. Can Med Assoc $J$ 179: 491. [Crossref]

3. Yaqub S, Mohkum S, Mukhtar KN (2013) Purple urine bag syndrome: A case report and review of literature. Indian J Nephrol 23:140-142. [Crossref]

Copyright: C2019 McDonnell D. This is an open-access article distributed under the terms of the Creative Commons Attribution License, which permits unrestricted use, distribution, and reproduction in any medium, provided the original author and source are credited. 